\title{
Cost-effectiveness of intrapleural use of tissue plasminogen activator and DNase in pleural infection: evidence from the MIST2 randomised controlled trial
}

\author{
Ramon Luengo-Fernandez (1) 1 , Erika Penz², Melissa Dobson³, \\ loannis Psallidas ${ }^{3}$, Andrew J. Nunn ${ }^{4}$, Nick A. Maskell ${ }^{5}$ and Najib M. Rahman ${ }^{3,6}$ \\ Affiliations: ${ }^{1}$ Health Economics Research Centre, Nuffield Dept of Population Health, University of Oxford, \\ Oxford, UK. ${ }^{2}$ Dept of Medicine, University of Saskatchewan, Saskatoon, SK, Canada. ${ }^{3}$ Oxford Respiratory Trials \\ Unit, Nuffield Dept of Clinical Medicine, University of Oxford, Oxford, UK. ${ }^{4}$ MRC Clinical Trials Unit, University \\ College London, London, UK. ${ }^{5}$ Academic Respiratory Unit, Bristol Medical School, University of Bristol, \\ Bristol, UK. ${ }^{6}$ Oxford NIHR Biomedical Research Centre, Oxford, UK.
}

Correspondence: Najib M. Rahman, Oxford Respiratory Trials Unit, Nuffield Dept of Clinical Medicine, University of Oxford, Oxford, OX3 7LE, UK. E-mail: najib.rahmanandm.ox.ac.uk

@ERSpublications

The MIST2 trial showed that combined intrapleural use of t-PA and DNase was effective when compared with single agents or placebo in the treatment of pleural infection. This economic evaluation shows that $\mathrm{t}-\mathrm{PA}-\mathrm{DNa}$ e is likely to be highly cost-effective. bit.ly/2vYZhWt

Cite this article as: Luengo-Fernandez R, Penz E, Dobson M, et al. Cost-effectiveness of intrapleural use of tissue plasminogen activator and DNase in pleural infection: evidence from the MIST2 randomised controlled trial. Eur Respir J 2019; 54: 1801550 [https://doi.org/10.1183/13993003.01550-2018].

ABSTRACT The MIST2 (Second Multicentre Intrapleural Sepsis Trial) trial showed that combined intrapleural use of tissue plasminogen activator ( $\mathrm{t}-\mathrm{PA}$ ) and recombinant human DNase was effective when compared with single agents or placebo. However, the treatment costs are significant and overall costeffectiveness of combined therapy remains unclear.

An economic evaluation of the MIST2 trial was performed to assess the cost-effectiveness of combined therapy. Costs included were those related to study medications, initial hospital stay and subsequent hospitalisations. Outcomes were measured in terms of life-years gained. All costs were reported in euro and in 2016 prices.

Mean annual costs were lowest in the t-PA-DNase group (EUR 10605 for t-PA, EUR 17856 for DNase, EUR 13483 for placebo and EUR 7248 for t-PA-DNase; $\mathrm{p}=0.209$ ). Mean 1-year life expectancy was 0.988 for t-PA, 0.923 for DNase, and 0.969 for both placebo and t-PA-DNase $(\mathrm{p}=0.296)$. Both DNase and placebo were less effective, in terms of life-years gained, and more costly than t-PA. When placebo was compared with t-PA-DNase, the incremental cost per life-year gained of placebo was EUR 1.6 billion, with a probability of 0.85 of t-PA-DNase being cost-effective.

This study demonstrates that combined t-PA-DNase is likely to be highly cost-effective. In light of this evidence, a definitive trial designed to facilitate a thorough economic evaluation is warranted to provide further evidence on the cost-effectiveness of this promising combined intervention.

\footnotetext{
This article has supplementary material available from erj.ersjournals.com

Clinical trial: The MIST2 trial on which this study is based was registered at the ISRCTN registry with identifier ISRCTN57454527. No individual patient data will be made available. The study protocol is available on request.
} 


\section{Introduction}

Pleural infection is a common and highly morbid condition. The incidence is increasing in both children and adults [1-5], and outcomes remain poor with up to $20 \%$ mortality at 1 year and failure of medical therapy in up to $30 \%$ of cases [6-10]. Median hospital stay is between 12 and 15 days $[6,7,9,10]$, and we have previously estimated this condition as costing EUR 4223 per patient [7]. Interventions to improve drainage, reduce infection and improve outcomes such as need for surgery and time in hospital are therefore priorities in care [8].

The MIST2 (Second Multicentre Intrapleural Sepsis Trial) trial was a double-blind, double-dummy, randomised placebo controlled trial assessing the combination of intrapleural tissue plasminogen activator (t-PA) and recombinant human DNase as an adjunct to drainage in patients with pleural infection. This study demonstrated significantly improved fluid drainage (measured radiologically) when compared with the single use of medications and placebo [7]. In secondary outcomes, MIST2 demonstrated t-PA-DNase therapy reduced the frequency of surgical referral (OR 0.17; $\mathrm{p}=0.03)$ and shortened length of stay in hospital.

Since publication of the MIST2 trial, there have been numerous case series of the use of t-PA-DNase as both "rescue therapy" and as an alternative to surgery in selected patients, totalling over 500 patients to date [11-20]. However, the costs of t-PA and DNase given twice daily for 3 days (as per the MIST2 protocol) are considerable (British National Formulary: www.bnf.org). It is not as yet clear if these increased medication costs are offset by reductions in surgical referral and shortened length of hospital stay.

This study was therefore conducted to specifically address whether use of t-PA-DNase therapy is cost-effective compared with individual use of DNase, t-PA and placebo, using the original data from the MIST2 trial.

\section{Methods}

\section{Patients}

Eligibility criteria were clinical evidence of infection and pleural fluid that was macroscopically purulent, positive on culture for bacterial infection, or positive for bacteria on Gram staining, or pleural fluid that had a $\mathrm{pH}<7.2$ (measured by means of a blood gas analyser). Evidence of infection, which was assessed by the recruiting physician, included the presence of fever and elevated serum levels of inflammatory markers such as C-reactive protein or an elevated white cell count.

\section{Study design}

MIST2 was a double-blind, double-dummy, factorial randomised trial conducted at 11 centres in the UK between December 2005 and November 2008 (ISRCTN registry identifier ISRCTN57454527) [7]. As per the study protocol, patients who did not receive any of the study medications and had pleural opacity at baseline that was $<5 \%$ of the hemithorax area on chest radiography were excluded. A total of 210 adult patients were enrolled into the study and randomised: 55 received double placebo, $52 \mathrm{t}$-PA, 51 DNase, and $52 \mathrm{t}-\mathrm{PA}$ and DNase. Patients were then followed-up for a period of 12 months. The dose of DNase was $5 \mathrm{mg}$ and the dose of $\mathrm{t}-\mathrm{PA}$ was $10 \mathrm{mg}$. Intrapleural medications were each given twice daily for 3 days and each administration was followed by clamping of the drain to permit the study drug to remain in the pleural space for $1 \mathrm{~h}$.

\section{Assessments}

The perspective adopted in the economic analysis was that of the hospital provider, with only the direct healthcare costs associated with initial hospitalisation, surgery and subsequent hospitalisation over the 12-month follow-up period included. All costs were reported in 2016 prices. British pounds were converted to euro (GBP 1=EUR 0.877; http://ec.europa.eu/Eurostat).

Initial hospitalisation length of stay was estimated using information from patients' trial records. This was defined as the time between the date of randomisation and discharge to home or to a nursing/residential care home. For patients who required thoracic surgery, duration of time in a surgical ward was estimated as the time between the date of surgery and the date of discharge from surgery. Unit costs were obtained from NHS Reference Costs [21]. A day in hospital was valued using the weighted daily average for Healthcare Resource Groups (HRGs) for "Lung Abscess and Empyema with Interventions", which was then multiplied to the patient's length of stay. For patients requiring thoracic surgery, a day in hospital was valued using the weighted daily averages of the three elective and three nonelective HRGs for "Major Thoracic Procedures, 19 years and over". Costs relating to admissions where patients underwent surgical procedures included both the cost of the hospital stay and of the procedures captured under that HRG. 
Information on subsequent hospitalisations over the 12-month follow-up period was obtained from patients' trial records. For each hospitalisation, information on the date of admission and discharge and the reason for that admission was recorded. Reasons for admission were translated into International Statistical Classification of Diseases and Related Health Problems 10th Revision codes and Office of Population Censuses and Surveys Classification of Surgical Operations and Procedures version 4 codes, which in turn were converted into an HRG using the HRG4+ Reference Cost Grouper (NHS Information Centre). HRGs were then valued using NHS Reference Costs [21]. Unit costs are reported in table 1. Medication costs were obtained from the British National Formulary. Total medication costs for each patient were then estimated: for t-PA (alteplase) EUR $164 \times$ twice daily $\times 3$ days and for DNase (Pulmozyme) EUR $19 \times$ two doses of $2.5 \mathrm{mg} \times$ twice daily $\times 3$ days.

In the absence of prospectively collected health-related quality of life, which would have enabled the estimation of quality-adjusted life-years gained, we evaluated the impact of the interventions on life-years gained. Life-years gained were defined in this study as the number of days a patient survived during the year after they were randomised in the study divided by 365.25 days. Therefore, a value of 1 life-year gained was assigned for surviving patients.

\section{Statistical analysis}

A within-trial economic analysis was undertaken, with total healthcare costs and life-years gained per patient calculated for the 12 months of the trial period in each of the four groups. Given the timeframe of the analysis as 1 year, discounting of costs and benefits was not performed. All analyses were carried out on an intention-to-treat basis using Stata/MP version 13 (StataCorp, College Station, TX, USA). Length of stay in hospital, costs and life-years gained are reported as means with standard deviations, with differences across the four groups compared using ANOVA. Statistical significance was considered at $\mathrm{p}<0.050$.

Treatment with chest tube drainage, antibiotics and saline flushes is current practice in the UK according to evidence-based guidelines (i.e. comparable to placebo in MIST2) [8]. As a result, we first compared each of the three trial interventions with placebo. To assess cost-effectiveness we estimated the incremental cost-effectiveness ratio (ICER), undertaken by dividing the mean cost difference between the placebo and the intervention by the difference in mean life-years gained.

In addition, an incremental analysis was conducted, rank ordering each intervention in terms of total costs. The mean cost difference between the second least costly intervention and the least costly intervention divided by the difference in mean life-years gained for these two interventions was used to estimate the ICER. Analysis was then repeated in increasing order of cost. 95\% confidence intervals were derived for the mean cost and life-years gained differences between the groups using nonparametric bootstrap sampling with 1000 replications. To assess the probability that an intervention was cost-effective at different willingness to pay thresholds for an additional life-year gained, cost-effectiveness acceptability curves were used with 1000 bootstrap estimates of mean costs and life-years gained for each of the four interventions [22]. An intervention was deemed cost-effective if the additional cost per life-year gained was below GBP 30000 (EUR 34220) [23].

A series of one-way sensitivity analyses was then performed. As there were a large number of different reasons why patients were readmitted into hospital, with each of these reasons varying substantially in

\begin{tabular}{|c|c|c|}
\hline & Unit cost EUR & Source \\
\hline \multicolumn{3}{|c|}{ Study medications, per dose } \\
\hline Alteplase 10 mg & 164 & British National Formulary \\
\hline Pulmozyme $2.5 \mathrm{mg}$ & 19 & British National Formulary \\
\hline \multicolumn{3}{|c|}{ Initial hospital stays, per day } \\
\hline Initial stay, nonsurgery & 502 & NHS Reference Costs \\
\hline Initial stay, surgery & 955 & NHS Reference Costs \\
\hline \multicolumn{3}{|l|}{ Subsequent admissions } \\
\hline Hospital stay ${ }^{\#}$ & $756^{\#}$ & NHS Reference Costs \\
\hline
\end{tabular}


cost, rather than applying cause-specific unit costs, we applied the weighted average unit cost reported in table 1 . As costs of trial medications are likely to vary considerably both over time (e.g. introduction of generic versions) and across countries, we varied the costs of medications by reducing their cost by $50 \%$ and increasing their cost by $100 \%$.

\section{Results}

A total of 210 adult patients were enrolled into the study and randomised: 55 received double placebo, 52 t-PA, 51 DNase, and $52 \mathrm{t}-\mathrm{PA}$ and DNase. However, there was missing length of stay and subsequent resource use in 32 patients (seven receiving double placebo, eight t-PA, 10 DNase, and seven t-PA and DNase). As a result, this analysis is based on the 178 patients having complete resource use: 48 receiving double placebo; 44 t-PA, 41 DNase, and 45 t-PA and DNase.

\section{Resource use, costs and life-years gained}

There were no significant differences across the four groups in terms of initial hospital stays and total number of days in hospital over the 12 -month period ( $p=0.265$ and $p=0.273$, respectively). However, although not statistically significant, the mean number of days in hospital was lower for the t-PA-DNase group, in terms of initial hospital stays (both surgical and nonsurgical) and hospital readmissions (table 2).

Except for trial medications, patients randomised to t-PA-DNase had the lowest levels of hospital care costs (table 3). Over the 12-month follow-up period, and after including the costs of medications, patients randomised to t-PA-DNase had total costs of EUR $7248 \pm 4922$ compared with EUR $10605 \pm 15413$ for t-PA, EUR $17856 \pm 34861$ for DNase and EUR $13483 \pm 28798$ for placebo. Although differences in total costs between the four patient groups were not statistically significant $(p=0.209)$, patients in the $t$-PADNase group incurred significantly lower costs than patients randomised to DNase $(p=0.041)$. Patients randomised to t-PA had the highest number of life-years gained $(0.988 \pm 0.081$ life-years gained), whereas patients randomised to DNase had the lowest number of life-years gained $(0.923 \pm 0.228$ life-years gained). However, differences in 1-year life expectancy were not statistically significant across patient groups $(\mathrm{p}=0.296)$.

\section{Cost-effectiveness: placebo versus trial interventions}

Given that placebo is currently standard UK practice in the form of saline flushes, we individually compared placebo with each of the three interventions in the trial (table 3). Placebo was found to be dominant over DNase (i.e. it was both more effective and less costly), whereas it was dominated by t-PA (i.e. placebo was less effective and more costly). When placebo was compared with t-PA-DNase the additional cost per life-year gained was EUR 1.6 billion. In this comparison, the probability that t-PADNase was cost-effective at a GBP 30000 (EUR 34220) cost per life-year gained threshold was 0.96.

Results of the sensitivity analysis showed that varying trial medication costs (reduction of 50\% and increase of $100 \%$ ) had no impact on cost-effectiveness. Using the overall mean-weighted unit cost to value subsequent days in hospital, rather than cause-specific unit costs, did not impact cost-effectiveness (supplementary material).

When we compared all the interventions in an incremental analysis, we found that DNase and placebo were less effective, in terms of life-years gained, and more costly than t-PA. As a result, for the incremental cost-effectiveness analysis t-PA was compared with t-PA-DNase, the least costly intervention (table 4).

TABLE 2 Number of days in hospital over the 12-month follow-up period

\begin{tabular}{|c|c|c|c|c|}
\hline & $t-P A$ & DNase & Placebo & t-PA-DNase \\
\hline Subjects & 44 & 41 & 48 & 45 \\
\hline \multicolumn{5}{|l|}{ Initial hospital stay } \\
\hline Number of days, surgical & $2.05 \pm 9.30$ & $5.66 \pm 18.07$ & $1.17 \pm 4.36$ & $0.24 \pm 1.15$ \\
\hline Number of days, total & $16.52 \pm 22.79$ & $28.24 \pm 61.41$ & $24.81 \pm 56.11$ & $11.78 \pm 9.43$ \\
\hline \multicolumn{5}{|l|}{ Subsequent hospital stays } \\
\hline \multicolumn{5}{|l|}{ Total hospital stays } \\
\hline Number of days & $17.27 \pm 24.79$ & $29.00 \pm 62.01$ & $26.19 \pm 56.66$ & $11.93 \pm 9.57$ \\
\hline
\end{tabular}

Data are presented as $n$, mean \pm SD or $n(\%)$. t-PA: tissue plasminogen activator. 
TABLE 3 Mean costs and outcomes over the 12-month follow-up period

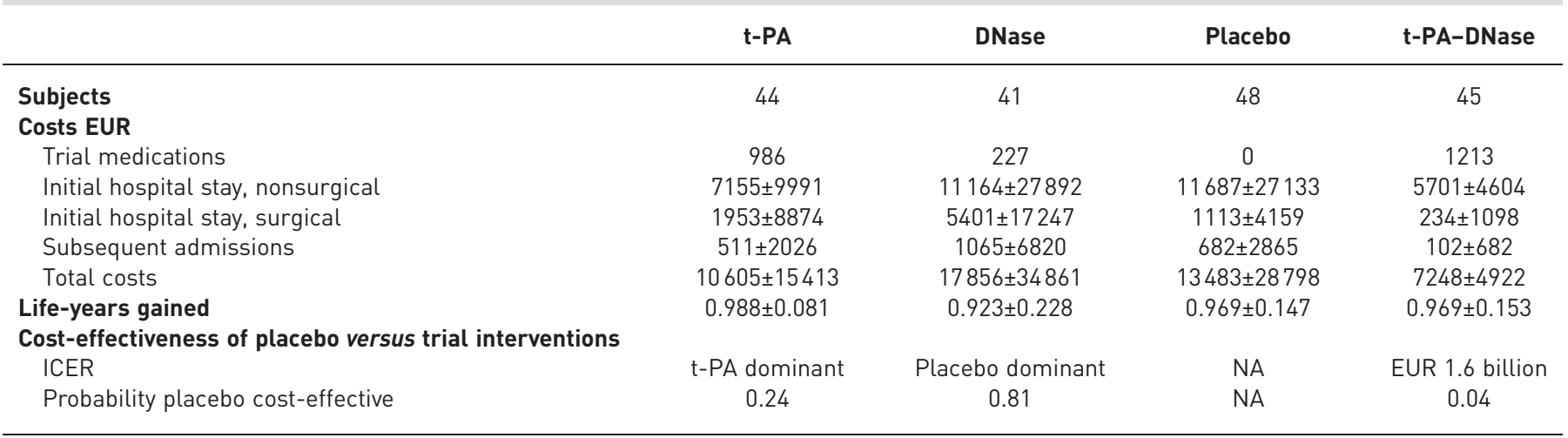

Data are presented as $n$ or mean \pm SD. t-PA: tissue plasminogen activator; ICER: incremental cost-effectiveness ratio; NA: not applicable.

When compared with t-PA-DNase, the incremental cost per life-year gained was EUR 178166 . This is much higher than the currently recommended willingness to pay thresholds recommended by the National Institute for Health and Care Excellence [23]. At a threshold of GBP 30000 (EUR 34220) per life-year gained, the probability that t-PA-DNase was cost-effective was 0.86 , whereas for t-PA this was 0.12. Placebo and DNase had a probability of less than 0.05 of being cost-effective. As shown in figure 1 , at any willingness to pay threshold for an additional life-year gained ranging between GBP 0 and GBP 100000 (EUR 114000) the probability of t-PA-DNase being cost-effective remained above 0.50 . For placebo and DNase the probability of being cost-effective never exceeded 0.10 .

\section{Discussion}

Using costs collected alongside a randomised clinical trial evaluating intrapleural therapy for empyema over a 1-year period, we found that administration of t-PA-DNase given twice daily for 3 days was most cost-effective compared with t-PA alone, DNase alone or placebo. Despite the added medication costs associated with t-PA and DNase, overall costs were lower in the combined treatment group than with individual therapies alone, highlighting the benefit seen in the original clinical trial in terms of reduced length of stay in hospital and surgical interventions.

This finding is clinically important with potential impact on current treatment. Although the MIST2 regimen is currently used only in patients who are failing medical therapy for pleural infection, and may not have a surgical option in most hospitals, these data suggest that it may be cost-effective to treat patients with pleural infections with a combination of t-PA and DNase early in treatment. The MIST2 trial recruited all patients with pleural infection and began treatment as soon as possible on admission; therefore, this study provides some economic rationale as to the use of the MIST2 regimen in all such cases. Our results suggest that using t-PA-DNase in preference to standard care (saline flushes, which is equivalent to placebo) in patients with pleural infection might save EUR 5700 per patient treated.

There are limitations to this analysis. First, the economic evaluation was conducted retrospectively and not concurrently alongside the clinical trial. The number of patients in each trial group was small, with the trial not being designed to detect differences in healthcare costs between the groups. Relevant healthcare resource use categories such as use of accident and emergency services, critical care, and outpatient and primary care were not evaluated. Furthermore, the timeframe of our analysis is $<1$ year and therefore cost-effectiveness of t-PA-DNase beyond this period is uncertain. However, given the acute nature of

TABLE 4 Cost-effectiveness of treatments for pleural infection at 12 months

Incremental costs EUR $(95 \% \mathrm{CI})$

\section{Incremental life-years} $(95 \% \mathrm{Cl})$

\section{Incremental cost-effectiveness ratio}

\section{Probability of intervention} being cost-effective

\begin{tabular}{lccc}
\hline t-PA-DNase & & & \\
t-PA & $3357(-335-8114)$ & $0.019(-0.025-0.062)$ & EUR 178166 \\
Placebo & $2878(-4280-10874)$ & $-0.019(-0.061-0.020)$ & t-PA both more effective and less costly \\
DNase & $4373(-6700-15940)$ & $-0.045(-0.116-0.016)$ & t-PA both more effective and less costly \\
\hline
\end{tabular}

t-PA: tissue plasminogen activator. ${ }^{\#}$ : assuming a cost-effectiveness threshold of GBP 30000 (EUR 34220 ) per life-year gained. 


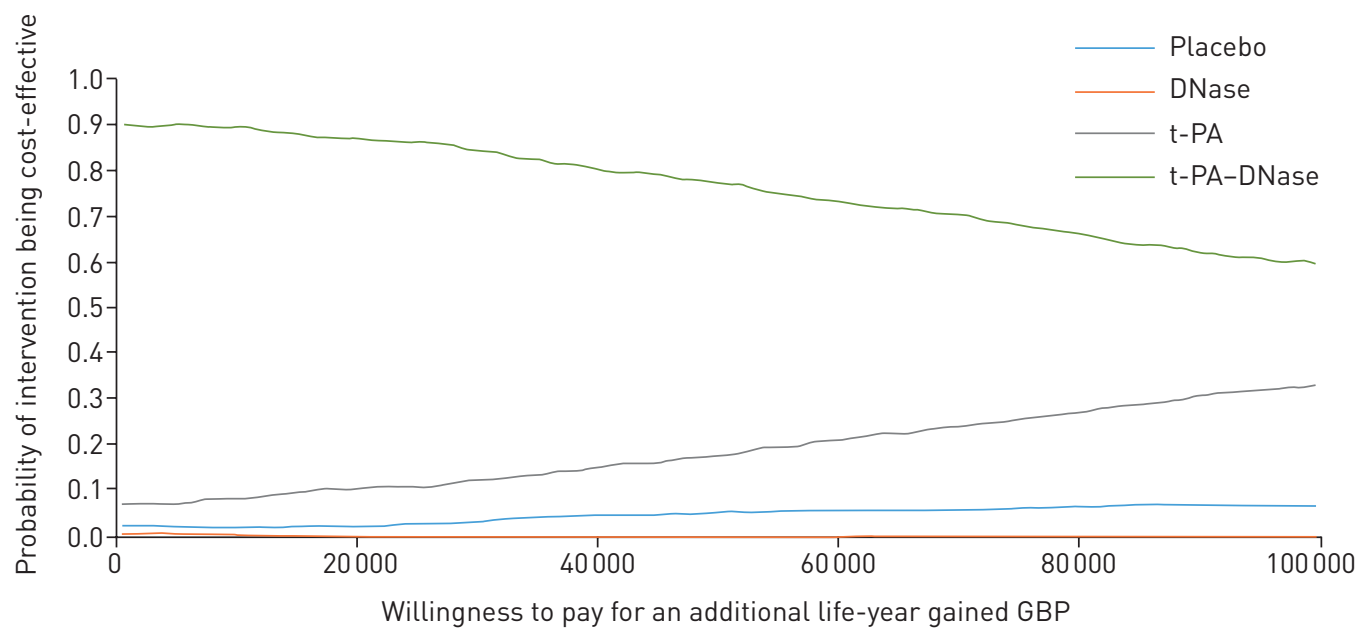

FIGURE 1 Cost-effectiveness acceptability curve. t-PA: tissue plasminogen activator.

empyema and its general treatment, it is reasonable to expect that all relevant hospitalisations and costs would have been captured in the 1-year follow-up period. Prospective follow-up data from randomised and observational studies suggest that the majority of outcomes occur within 3 months and all within 12 months in pleural infection $[6,7,9,10]$.

Quality of life via patient questionnaires was not assessed in this trial and therefore the health outcome expressed in our analysis was life-years gained. Ideally, in order to assist decision makers in choosing among different healthcare interventions, costs per quality-adjusted life-years are typically recommended [6]. However, given that combined treatment not only reduced hospitalisation stays over the 1-year follow-up period but also reduced surgical interventions, it is likely that quality of life would also be higher in the combined treatment group, hence improving the cost-effectiveness of the intervention.

Finally, the MIST2 trial was conducted in the UK, using British unit costs to value hospital resource use and medications. Therefore, the results presented will be most generalisable to UK settings. However, we do believe that our measures of resource use, showing the potential for combined t-PA-DNase treatment to considerably reduce overall hospital resource use, are likely to be applicable to other jurisdictions.

Previous evidence from the MIST2 trial showed that combination treatment with t-PA and DNase was effective in improving fluid drainage in patients with pleural infection [1]. This study now highlights that combined treatment is also likely to be highly cost-effective. In light of this evidence, a definitive trial designed to facilitate a thorough economic evaluation is therefore warranted to provide further evidence about the cost-effectiveness of this promising combined intervention in order to assist decision makers.

Author contributions: R. Luengo-Fernandez performed the health economics analysis and wrote the first draft of the manuscript. E. Penz helped write the first draft of the manuscript and provided intellectual input for data interpretation. M. Dobson, I. Psallidas, A.J. Nunn, N.A. Maskell and N.M. Rahman were involved in either original data collection, subsequent intellectual input or manuscript writing All authors reviewed and approved the final manuscript.

Conflict of interest: R. Luengo-Fernandez has nothing to disclose. E. Penz reports personal fees (participation in advisory boards) from AstraZeneca, GlaxoSmithKline and Boehringer Ingelheim, outside the submitted work. M. Dobson has nothing to disclose. I. Psallidas works as a Medical Science Director in AstraZeneca in a different scientific area not relevant to the article. A.J. Nunn reports an unrestricted educational grant from Roche UK, during the conduct of the study. N.A. Maskell has nothing to disclose. N.M. Rahman reports an unrestricted educational grant from Roche UK, during the conduct of the study.

Support statement: This work had no specific funding. The original trial was partly funded by an unrestricted educational grant from Roche UK and by others. A.J. Nunn's salary came through core funding MC_UU_12023/27 Tuberculosis Treatment Trials.

\section{References}

1 Grijalva CG, Zhu Y, Pekka NJ, et al. Emergence of parapneumonic empyema in the USA. Thorax 2011; 66: 663-668.

2 Desrumaux A, Francois P, Pascal C, et al. Epidemiology and clinical characteristics of childhood parapneumonic empyemas. Arch Pediatr 2007; 14: 1298-1303.

3 Farjah F, Symons RG, Krishnadasan B, et al. Management of pleural space infections: a population-based analysis. J Thorac Cardiovasc Surg 2007; 133: 346-351. 

2007; 52: 25-27.

5 Finley C, Clifton J, Fitzgerald JM, et al. Empyema: an increasing concern in Canada. Can Respir J 2008; 15: 85-89.

6 Maskell NA, Davies CW, Nunn AJ, et al. U.K. controlled trial of intrapleural streptokinase for pleural infection. N Engl J Med 2005; 352: 865-874.

7 Rahman NN, Maskell NA, West A, et al. Intrapleural use of tissue plasminogen activator and DNase in pleural infection. N Engl J Med 2011; 365: 518-526.

8 Davies HE, Davies RJ, Davies CW. Management of pleural infection in adults: British Thoracic Society Pleural Disease Guideline 2010. Thorax 2010; 65: Suppl. 2, ii41-ii53.

9 Davies CW, Kearney SE, Gleeson FV, et al. Predictors of outcome and long-term survival in patients with pleural infection. Am J Respir Crit Care Med 1999; 160: 1682-1687.

10 Ferguson AD, Prescott RJ, Selkon JB, et al. The clinical course and management of thoracic empyema. QJM 1996; 89: 285-289.

11 Majid A, Ochoa S, Chatterji S, et al. Safety and efficacy of tissue plasminogen activator and DNase for complicated pleural effusions secondary to abdominal pathology. Ann Am Thorac Soc 2017; 14: 342-346.

12 Bishwakarma R, Shah S, Frank L, et al. Mixing it up: coadministration of tPA/DNase in complicated parapneumonic pleural effusions and empyema. J Bronchology Interv Pulmonol 2017; 24: 40-47.

13 Majid A, Kheir F, Folch A, et al. Concurrent intrapleural instillation of tissue plasminogen activator and DNase for pleural infection. A single-center experience. Ann Am Thorac Soc 2016; 13: 1512-1518.

14 Sharan LA, Price TP, Hehn B, et al. A 22-year-old man with pleural tuberculosis associated hydropneumothorax: case report and literature review. Respir Med Case Rep 2016; 18: 27-30.

15 Mehta HJ, Biswas A, Penley AM, et al. Management of intrapleural sepsis with once daily use of tissue plasminogen activator and deoxyribonuclease. Respiration 2016; 91: 101-106.

16 Croft DP, Philippo SM, Prasad P. A case of Lemierre's syndrome with septic shock and complicated parapneumonic effusions requiring intrapleural fibrinolysis. Respir Med Case Rep 2015; 16: 86-88.

17 Popowicz N, Nash M, Lee YC. Unintentional intramuscular administration of tPA/DNase for pleural infection. Respirol Case Rep 2014; 2: 144-146.

18 Popowicz N, Piccolo F, Shrestha R, et al. Two sequential tPA/DNase courses for noncommunicating loculated collections in pleural infection. Respirol Case Rep 2014; 2: 87-89.

19 Piccolo F, Pitman N, Bhatnagar R, et al. Intrapleural tissue plasminogen activator and deoxyribonuclease for pleural infection. An effective and safe alternative to surgery. Ann Am Thorac Soc 2014; 11: 1419-1425.

20 Porcel JM. Minimally invasive treatment of complicated parapneumonic effusions and empyemas in adults. Clin Respir J 2018; 12: 1361-1366.

21 Department of Health. NHS Reference Costs 2015 to 2016. 2016. www.gov.uk/government/publications/ nhs-reference-costs-2015-to-2016 Last date accessed: March 28, 2017.

22 Fenwick E, Byford S. A guide to cost-effectiveness acceptability curves. Br J Psychiatry 2005; 187: 106-108.

23 National Institute for Health and Care Excellence. Guide to the Methods of Technology Appraisal 2013. London, NICE, 2013. 